INPLASY

PROTOCOL

To cite: Zhang et al.

Association between

acupuncture and grade 1

hypertension a systematic

review and meta-analysis.

Inplasy protocol 202130071.

doi:

10.37766/inplasy2021.3.0071

Received: 19 March 2021

Published: 20 March 2021

Corresponding author:

MuXin Zhang

1157743771@qq.com

Author Affiliation:

ShanDong university of

traditional Chinese medicine

Support: Shandong Provincial

Health Commission Integrated

Traditional Chinese and Western

Medicine Prevention and

Treatment of Common and

Frequently-occurring Diseases

2019-11.

Review Stage at time of this

submission: Data extraction.

Conflicts of interest:

None declared.

\section{Association between acupuncture and grade 1 hypertension a systematic review and meta-analysis}

Review question / Objective: (1) P: Patients with grade 1 hypertension. (2) I: Acupuncture. (3) C: Western medicine treatment alone, lifestyle adjustment, placebo or no treatment. (4) O: BP value after treatment, Pre-post BP change value, response rate of treatment, adverse effects.

Condition being studied: We have completed the preliminary data retrieval, study selection, and formally screened the search results according to the qualification criteria. Now we are doing data extraction.

Information sources: Ebase, PubMed, Chorane, China National Knowledge Infrastructure (CNKI), Wan Fang database.

INPLASY registration number: This protocol was registered with the International Platform of Registered Systematic Review and Meta-Analysis Protocols (INPLASY) on 20 March 2021 and was last updated on 20 March 2021 (registration number INPLASY202130071).

\section{INTRODUCTION}

Review question / Objective: (1) P: Patients with grade 1 hypertension. (2) I:
Acupuncture. (3) C: Western medicine treatment alone, lifestyle adjustment, placebo or no treatment. (4) $\mathrm{O}$ : $\mathrm{BP}$ value after treatment, Pre-post BP change value, 
response rate of treatment, adverse effects.

Condition being studied: We have completed the preliminary data retrieval, study selection, and formally screened the search results according to the qualification criteria. Now we are doing data extraction.

\section{METHODS}

Participant or population: Patients with grade 1 hypertension.

Intervention: Acupuncture.

Comparator: Western medicine treatment alone, lifestyle adjustment, placebo or no treatment.

Study designs to be included: Randomized controlled trials of acupuncture in the treatment of hypertension.

Eligibility criteria: (1) Duplicate literatures in the test population. (2) Researches on case studies, animal experiments, and ethical exploration. (3) Comparisons of different acupuncture methods or different acupoints. (4) Literatures of participants suffering from mental illnesses and other diseases (5) The blood pressure data unit cannot be converted to $\mathrm{mmHg}$. (6) Simple randomized controlled trials, semirandomized controlled trials and selfcontrolled trials. (7) The results reported in the literature are controversial.

Information sources: Ebase, PubMed, Chorane, China National Knowledge Infrastructure (CNKI), Wan Fang database.

Main outcome(s): BP value after treatment, Pre-post BP change value, response rate of treatment, adverse effects.

Quality assessment / Risk of bias analysis: (1) random sequence generation (selection bias); (2) allocation concealment (selection bias); (3) blinding of participants and personnel (performance bias); (4) blinding of outcome assessment (detection bias); (5) incomplete outcome data (attrition bias); (6) selective reporting (reporting bias); and (7) any other bias.

Strategy of data synthesis: Data were assembled and analyzed using Review Manager (RevMan) Version 5.3 (The Nordic Cochrane Center, Copenhagen, Denmark) and the Statistical Package for the Social Sciences (SPSS) V.19.0 (SPSS Inc, Chicago, Illinois, USA). Continuous data were presented as mean differences (MDs) with 95\% confidence interval $(\mathrm{Cl})$, dichotomous data were presented as relative risk (RR) with $95 \% \mathrm{Cl}$. We used 12 statistic to check for heterogeneity, and defined $12>50 \%$ as indicating significant heterogeneity. If there was no significant heterogeneity, the fixed effects model was used; if there was significant heterogeneity, the random effects model was used. If the heterogeneity was significant, the possible causes would be investigated from a clinical perspective through subgroup analysis. According to the sample size, age, gender, type of intervention, treatment period, etc., subgroup analysis was performed. Funnel plots were used to detect publication bias.

Subgroup analysis: The subgroup analysis was performed independently according to the setting of the control group and the duration of treatment.

Sensitivity analysis: For the highly heterogeneous outcome indicators, relevant sensitivity analysis was carried out.

Country(ies) involved: China.

Keywords: acupuncture, grade 1 hypertension, meta-analyses, randomized controlled trial

Contributions of each author:

Author 1 - MuXin Zhang.

Email: 1157743771@qq.com

Author 2 - YuShuo Zhu.

Email: 957712401@qq.com

Author 3 - YunLun Li.

Email: li.yunlun@163.com

Author 4 - Zhen Hua.

Email: huazhen0326@163.com 